\title{
Short-Term and Long-Term Effects of Levetiracetam Monotherapy on Homocysteine Metabolism in Children with Epilepsy: A Prospective Study
}

\author{
Achilleas Attilakos ${ }^{a}$ \\ Maria Paschalidou ${ }^{a}$ \\ Anastasia Garoufi \\ Maria Tsirouda ${ }^{a}$ \\ Anna Papadopoulou ${ }^{a}$ \\ Maria Karalexi $^{\mathrm{a}}$ \\ Argirios Dinopoulos ${ }^{a}$ \\ aThird Department of Pediatrics, \\ National and Kapodistrian \\ University of Athens, "Attikon" Hospital, \\ Athens, Greece \\ becond Department of Pediatrics, \\ National and Kapodistrian \\ University of Athens, \\ "P\&A Kyriakou" Children's Hospital, \\ Athens, Greece
}

Background and Purpose Long-term treatment with some older antiepileptic drugs may lead to hyperhomocysteinemia. Levetiracetam (LEV) is a newer broad-spectrum antiepileptic agent whose effects on homocysteine concentrations remain unclear. The purpose of this study was to prospectively determine the short-term and long-term effects of LEV monotherapy on homocysteine metabolism in children with epilepsy.

Methods The study population consisted of 32 children [18 females, 14 males; age $5.94 \pm 4.10$ years (mean $\pm S D)$, age range 1-15 years] who received LEV monotherapy for new-onset epilepsy. Serum folate, serum vitamin $\mathrm{B}_{12}$, and plasma total homocysteine ( $\mathrm{p}$-tHcy) were measured before and at 2 months ( $n=32), 6$ months $(n=25)$, and 12 months $(n=18)$ of LEV monotherapy.

Results p-tHcy was significantly decreased at 2 months of treatment $(p=0.031)$. Furthermore, analysis of covariance showed statistically significant decreases in p-tHcy at 2 months ( $p=0.013)$ and 6 months $(p=0.015)$ of LEV treatment after controlling for age, sex, body mass index, and LEV dose. There were no significant alterations in the other parameters during the study. The drug doses were $18.1 \pm 7.1,20.1 \pm 9.2$, and $21.2 \pm 11.8 \mathrm{mg} / \mathrm{kg}$ at 2,6 , and 12 months of LEV treatment, respectively.

Conclusions In contrast with older antiepileptic drugs, long-term LEV monotherapy in children with epilepsy does not cause adverse alterations on homocysteine metabolism. Larger prospective studies are needed to definitively clarify whether LEV may be considered a safer alternative drug for preventing antiepileptic-drug-induced cardiovascular complications in adult life.

Key Words children, epilepsy, homocysteine, levetiracetam.
Received August 14, 2018

Revised October 1, 2018

Accepted October 1, 2018

\section{Correspondence}

Achilleas Attilakos, MD, PhD

Third Department of Pediatrics,

National and Kapodistrian

University of Athens,

"Attikon" Hospital,

30 Miltiadou Street, Glyfada,

Athens 16675, Greece

Tel +306972891212

Fax +302105832229

E-mail attilakos@hotmail.com

\section{INTRODUCTION}

Hyperhomocysteinemia has been associated with an increased risk of occlusive vascular disease, brain atrophy, and seizure aggravation in epileptic patients. ${ }^{1-4}$ Long-term treatment with older antiepileptic drugs may lead to hyperhomocysteinemia by affecting the blood concentrations of folate, vitamin $\mathrm{B}_{12}$, and vitamin $\mathrm{B}_{6}{ }^{1,2}$

Levetiracetam (LEV) is a new antiepileptic drug with favorable efficacy and low toxicity profile in epilepsy treatment. ${ }^{5}$ There are very limited and conflicting data on the effect of LEV monotherapy on fasting plasma total homocysteine (p-tHcy). Elevated or normal homocysteine levels after initiating LEV treatment have been found in a few adult studies. ${ }^{2}$ Recently, the only cross-sectional study in children found that the homocysteine concentration was increased in 12 children (age 11.6 \pm 1.9 years, mean \pm SD) after LEV treatment for a period of $2.20 \pm 0.45$ years. $^{6}$

The purpose of this study was to prospectively determine the effect of LEV monotherapy

(a) This is an Open Access article distributed under the terms of the Creative Commons Attribution Non-Commercial License (https://creativecommons.org/licenses/by-nc/4.0) which permits unrestricted non-commercial use, distribution, and reproduction in any medium, provided the original work is properly cited. 
on homocysteine metabolism in children with epilepsy. To our knowledge, this is the first study to prospectively investigate the homocysteine, folate, and vitamin $\mathrm{B}_{12}$ levels during LEV monotherapy in children with epilepsy.

\section{METHODS}

The study population consisted of 32 children (18 females, 14 males; age $5.94 \pm 4.10$ years, age range $1-15$ years) who were treated for new-onset epilepsy with LEV monotherapy. Twenty-four of the children suffered from a localization-related epilepsy, and eight from a generalized epilepsy. Dropouts due to the need for other antiepileptic treatment and missing data reduced the sample size to 25 patients at 6 months and 18 patients at 12 months of LEV treatment. LEV was prescribed at a daily dose of $10-35 \mathrm{mg} / \mathrm{kg}$. Apart from epilepsy, all patients had normal growth, normal liver and renal function, were not taking any other medication, and had not taken antiepileptic medication previously.

Serum folate (s-F), serum vitamin $B_{12}\left(s-B_{12}\right)$, and p-tHcy were measured before and at 2, 6, and 12 months of LEV monotherapy. A standardized age- and sex-specific growth reference was used to calculate the body mass index for-age standard deviation score (BMI-SDS) (http://www.quesgen. com/BMIPedsCalc.php).

Informed parental consent was obtained for each participant, and the study was approved by the Institutional Review Board of Attikon University Hospital (study number 8659/21, July 2011). A blood sample was obtained after 10-12 hours of fasting, between 8.00 and 10.00 a.m. Plasma and serum aliquots were rapidly separated and frozen to $-80^{\circ} \mathrm{C}$ until being analyzed. p-tHcy was measured using particle-enhanced immunonephelometry with the BN II and BN ProSpec systems (Dade Behring, Marburg, Germany). S-F and s- $\mathrm{B}_{12}$ concentrations were measured using an immunoassay analyzer (COBAS E 601, Roche Diagnostics, Indianapolis, IN, USA).

Data were analyzed using the SPSS (version 23.0, IBM Corp., Armonk, NY, USA). The Wilcoxon signed-ranks test was used to assess the significance of parameters at baseline and after treatment with LEV. Correlations among all the parameters and between the parameters and the LEV dose were assessed by using Spearman's correlation coefficient. A $p$ value $<0.05$ was considered statistically significant. A regression-based analysis of covariance (ANCOVA) was also performed to assess the potential effects of confounding factors such as age, sex, BMI-SDS, and LEV dose on the associations of parameters evaluated with LEV treatment. Post-hoc tests using Bonferroni correction were used to assess the potential for type I errors when performing multiple statistical tests. The multivariate analysis and Bonferroni correction were implemented using SAS (version 9, SAS Institute Inc., Cary, NC, USA).

\section{RESULTS}

During the 12-month study period, all patients exhibited an absolute response to the treatment, with 12 of them requiring a minimal dose titration (up to $20 \mathrm{mg} / \mathrm{kg}$ ) within the first month of therapy and 6 of them requiring a significant dose titration of up to $35 \mathrm{mg} / \mathrm{kg}$ within the first 2 months of treatment.

P-tHcy was significantly decreased at 2 months of LEV treatment $(p=0.031)$. There were no significant alterations in the other parameters evaluated during the study (Table 1). The ANCOVA revealed statistically significant decreases in p-tHcy at 2 months ( $p=0.013)$ and 6 months $(p=0.015)$ of LEV treatment after controlling for age, sex, BMI-SDS, and LEV dose (Table 2). Also, the multivariate analysis revealed that $\mathrm{p}$-tHcy was increased at 12 months $(p=0.002)$ of treatment (Table 2). Among the confounding factors, only age was found to be borderline significantly associated with the $\mathrm{p}$ tHcy increase at 12 months of treatment $(p=0.052)$, whereas there was no significant association with sex $(p=0.092)$ or LEV dose $(p=0.666)$ (Table 2). Applying post-hoc tests using Bonferroni correction produced no significant alterations in the findings.

The drug doses were 18.1 $\pm 7.1,20.1 \pm 9.2$, and $21.2 \pm 11.8$ $\mathrm{mg} / \mathrm{kg}$ at 2, 6, and 12 months of LEV treatment, respectively. No correlation was found between p-tHcy and the other parameters evaluated or between $\mathrm{p}$-tHcy and the drug dose during treatment.

Comparing p-tHcy in our group with the reference range of p-tHcy in children ${ }^{7,8}$ revealed that p-tHcy was within age-

Table 1. Changes in $\mathrm{p}-\mathrm{HH} c \mathrm{~s}, \mathrm{~s}-\mathrm{F}_{1} \mathrm{~s}-\mathrm{B}_{12}$, and BMI-SDS during LEV monotherapy

\begin{tabular}{lccccccc}
\hline \multicolumn{1}{c}{ Parameter } & Before treatment & After 2 months $(\boldsymbol{n = 3 2 )}$ & $\boldsymbol{p}$ & After $\mathbf{6}$ months $(\boldsymbol{n}=\mathbf{2 5})$ & $\boldsymbol{p}$ & After 12 months $(\boldsymbol{n}=\mathbf{1 8})$ & $\boldsymbol{p}$ \\
\hline $\mathrm{p}-\mathrm{tHcy}(\mu \mathrm{mol} / \mathrm{L})$ & $6.85 \pm 1.46$ & $6.29 \pm 1.55$ & $0.031^{*}$ & $6.46 \pm 1.65$ & 0.123 & $7.22 \pm 2.36$ & 0.325 \\
$\mathrm{~s}-\mathrm{B}_{12}(\mathrm{pg} / \mathrm{mL})$ & $709.0 \pm 276.4$ & $679.8 \pm 273.3$ & 0.199 & $703.5 \pm 310.8$ & 0.463 & $702.5 \pm 295.0$ & 0.075 \\
$\mathrm{~s}-\mathrm{F}(\mathrm{ng} / \mathrm{mL})$ & $14.33 \pm 5.89$ & $13.37 \pm 5.40$ & 0.679 & $12.83 \pm 5.64$ & 0.478 & $12.21 \pm 5.59$ \\
$\mathrm{BMI}-\mathrm{SDS}$ & $0.29 \pm 1.67$ & $0.41 \pm 1.54$ & 0.533 & $0.19 \pm 1.73$ & 0.135 & $0.17 \pm 1.56$ & 0.133 \\
\hline
\end{tabular}

Data are mean \pm SD values. $p$ indicates statistical differences between pretreatment and post treatment levels.

*Statistical significance $(p<0.05)$.

BMI-SDS: body mass index for-age standard deviation score, LEV: levetiracetam, p-tHcy: plasma total homocysteine, s- $B_{12}$ : serum vitamin $B_{12}, s-F$ : serum folate. 
Table 2. Results from the analysis of covariance with age, sex, and LEV dose as covariates

\begin{tabular}{lccc}
\hline Parameter & $\begin{array}{c}\text { After 2 } \\
\text { months }(\boldsymbol{p})\end{array}$ & $\begin{array}{c}\text { After 6 } \\
\text { months }(\boldsymbol{p})\end{array}$ & $\begin{array}{c}\text { After 12 } \\
\text { months }(\boldsymbol{p})\end{array}$ \\
\hline Age & 0.652 & 0.332 & 0.052 \\
Sex & 0.737 & 0.875 & 0.092 \\
LEV dose & 0.194 & 0.395 & 0.666 \\
p-tHcy & $0.013^{*}$ & $0.015^{*}$ & $0.002^{*}$ \\
\hline
\end{tabular}

*Statistical significance $(p<0.05)$.

LEV: levetiracetam, p-tHcy: plasma total homocysteine.

specific reference limits in all of the current participants.

\section{DISCUSSION}

This study found that LEV exerts a transient short-term effect on homocysteine metabolism in children with epilepsy. There were no significant alterations in s-F, s- $\mathrm{B}_{12}$, and BMISDS at 2, 6, and 12 months of LEV treatment.

The available data on the effect of LEV monotherapy on p-tHcy are very limited and conflicting. Belcastro et al. ${ }^{9}$ reported normal p-tHcy in 32 adults with epilepsy treated with LEV monotherapy for at least 6 months, while Kim et al. ${ }^{10}$ reported a significant elevation of homocysteine with unchanged s-F and $\mathrm{p}-\mathrm{B}_{12}$ levels in 40 adults with newly diagnosed epilepsy after 6 months of LEV treatment. A recent cross-sectional study found increased homocysteine concentrations in 12 children (age 11.6 \pm 1.9 years) after LEV treatment for a period of $2.20 \pm 0.45$ years. $^{6}$

Our multivariate analysis demonstrated early significant decreases in p-tHcy at 2 and 6 months of LEV treatment, whereas it was significantly increased at 12 months $(p=0.002)$. We cannot explain the unexpected and intriguing finding of p-tHcy being decreased at 2 and 6 months of LEV treatment. This short-term effect cannot be attributed to changes in s-F or $s-B_{12}$ levels, since they remained unchanged during the study. Furthermore, this short-term effect cannot be explained by a possible seasonal variation of $\mathrm{p}$-tHcy. ${ }^{11}$ Since the levels of vitamin $\mathrm{B}_{6}$ were not measured, further studies including also evaluation of this cofactor are needed to clarify this issue. On the other hand, the increase in p-tHcy at 12 months of treatment can be explained by the participants being older at that time point, ${ }^{7,8}$ since only age was found to be borderline significantly associated with the p-tHcy increase at 12 months of treatment.

BMI-SDS did not change significantly in our study population during the follow-up period. This is in accordance with other studies that have found LEV to be weight-neutral, in contrast with older antiepileptic drugs that may induce weight gain. ${ }^{2}$
This study was subject to some limitations; the small number of included patients and not measuring vitamin $\mathrm{B}_{6}$, which plays a role in homocysteine metabolism. Although the plasma levels of LEV were not measured, LEV has a very favorable pharmacokinetic profile, characterized by excellent oral absorption and bioavailability (>95\%) and linear kinetics. ${ }^{12}$ The advantages of the present study include its prospective design covering a 1-year period and the homogeneity of the study group.

In conclusion, this study found that, in contrast with older antiepileptic drugs, applying LEV monotherapy for up to 1 year to children with epilepsy does not adversely affect homocysteine metabolism. Larger prospective studies that include evaluations of other cardiovascular risk factors are needed to definitively clarify whether LEV may be considered a safer alternative drug for preventing antiepileptic drug-induced cardiovascular complications in adult life.

\section{Conflicts of Interest}

The authors have no potential conflicts of interest to disclose.

\section{REFERENCES}

1. Belcastro V, Striano P. Antiepileptic drugs, hyperhomocysteinemia and B-vitamins supplementation in patients with epilepsy. Epilepsy Res 2012;102:1-7.

2. Katsiki N, Mikhailidis DP, Nair DR. The effects of antiepileptic drugs on vascular risk factors: a narrative review. Seizure 2014;23:677-684.

3. Gorgone G, Caccamo D, Pisani LR, Currò M, Parisi G, Oteri G, et al. Hyperhomocysteinemia in patients with epilepsy: does it play a role in the pathogenesis of brain atrophy? A preliminary report. Epilepsia 2009; 50 Suppl 1:33-36.

4. Baldelli E, Leo G, Andreoli N, Fuxe K, Biagini G, Agnati LF. Homocysteine potentiates seizures and cell loss induced by pilocarpine treatment. Neuromolecular Med 2010;12:248-259.

5. Lyseng-Williamson KA. Levetiracetam: a review of its use in epilepsy. Drugs 2011;71:489-514.

6. El-Farahaty RM, El-Mitwalli A, Azzam H, Wasel Y, Elrakhawy MM, Hasaneen BM. Atherosclerotic effects of long-term old and new antiepileptic drugs monotherapy: a cross-sectional comparative study. $J$ Child Neurol 2015;30:451-457.

7. Papandreou D, Mavromichalis I, Makedou A, Rousso I, Arvanitidou M. Reference range of total serum homocysteine level and dietary indexes in healthy Greek schoolchildren aged 6-15 years. Br J Nutr 2006; 96:719-724.

8. Vilaseca MA, Moyano D, Ferrer I, Artuch R. Total homocysteine in pediatric patients. Clin Chem 1997;43:690-692.

9. Belcastro V, Striano P, Gorgone G, Costa C, Ciampa C, Caccamo D, et al. Hyperhomocysteinemia in epileptic patients on new antiepileptic drugs. Epilepsia 2010;51:274-279.

10. Kim DW, Lee SY, Shon YM, Kim JH. Effects of new antiepileptic drugs on circulatory markers for vascular risk in patients with newly diagnosed epilepsy. Epilepsia 2013;54:e146-e149.

11. McKinley MC, Strain JJ, McPartlin J, Scott JM, McNulty H. Plasma homocysteine is not subject to seasonal variation. Clin Chem 2001; 47:1430-1436.

12. Patsalos PN. Pharmacokinetic profile of levetiracetam: toward ideal characteristics. Pharmacol Ther 2000;85:77-85. 